\title{
Sex differences in thermal detection and thermal pain threshold and the thermal grill illusion: a psychophysical study in young volunteers
}

\author{
Beate Averbeck ${ }^{1,3^{*}}$ (D) Lena Seitz ${ }^{1}$ Florian P. Kolb ${ }^{1}$ and Dieter F. Kutz ${ }^{2}$
}

\begin{abstract}
Background: Sex-related differences in human thermal and pain sensitivity are the subject of controversial discussion. The goal of this study in a large number of subjects was to investigate sex differences in thermal and thermal pain perception and the thermal grill illusion (TGl) as a phenomenon reflecting crosstalk between the thermoreceptive and nociceptive systems. The thermal grill illusion is a sensation of strong, but not necessarily painful, heat often preceded by transient cold upon skin contact with spatially interlaced innocuous warm and cool stimuli.

Methods: The TGI was studied in a group of 78 female and 58 male undergraduate students and was evoked by placing the palm of the right hand on the thermal grill $\left(20 / 40{ }^{\circ} \mathrm{C}\right.$ interleaved stimulus). Sex-related thermal perception was investigated by a retrospective analysis of thermal detection and thermal pain threshold data that had been measured in student laboratory courses over 5 years (776 female and 476 male undergraduate students) using the method of quantitative sensory testing (QST). To analyse correlations between thermal pain sensitivity and the TGl, thermal pain threshold and the TGI were determined in a group of 20 female and 20 male undergraduate students.

Results: The TGI was more pronounced in females than males. Females were more sensitive with respect to thermal detection and thermal pain thresholds. Independent of sex, thermal detection thresholds were dependent on the baseline temperature with a specific progression of an optimum curve for cold detection threshold versus baseline temperature. The distribution of cold pain thresholds was multi-modal and sex-dependent. The more pronounced TGl in females correlated with higher cold sensitivity and cold pain sensitivity in females than in males.

Conclusions: Our finding that thermal detection threshold not only differs between the sexes but is also dependent on the baseline temperature reveals a complex processing of "cold" and "warm" inputs in thermal perception. The results of the TGl experiment support the assumption that sex differences in cold-related thermoreception are responsible for sex differences in the TGl.
\end{abstract}

Keywords: Sex differences, Psychophysics, Thermal thresholds, Thermal grill illusion, Thermal pain, Cold sensitivity, Cold receptors

\footnotetext{
* Correspondence: beate.averbeck@|rz.uni-muenchen.de; http://www.cell. physiol.med.uni-muenchen.de/index.html

'Department of Physiology, University of Munich, Munich, Germany

${ }^{3}$ Department of Physiology, Biomedical Center Munich (BMC), University of

Munich, Planegg-Martinsried, D-82152, Germany

Full list of author information is available at the end of the article
} 


\section{Background}

Evidence suggests women and men experience and report pain differently. The most pronounced sex differences have been found for heat pain, with females showing lower heat pain threshold, tolerating less thermal heat and perceiving hot temperatures as more painful and more unpleasant than males [1-6]. Sex differences in cold pain as well as in thermal non-painful sensation have been described more rarely in the literature and the existing studies report higher sensitivity of females compared with males $[2,4,7,8]$. Sex differences in the thermal grill illusion (TGI), a phenomenon reflecting crosstalk between the thermoreceptive and nociceptive systems, have not been investigated so far.

The TGI, first reported by Thunberg in 1886, is generated by pairing innocuous warm and cold temperatures. This leads to a sensation of strong, but not necessarily painful, heat often preceded by transient cold [9]. Several studies indicate that the TGI is a very complex phenomenon that is generated by central higher order processing and reveals a relationship between the thermoreceptive and nociceptive systems [10-23]. Thus, sex differences in thermoreception and thermal pain perception may be related to sex differences in the TGI.

The aim of the present study was to determine sex differences in the TGI by testing 136 undergraduate medical students. We recorded qualities and intensities of sensation evoked by a $20 / 40{ }^{\circ} \mathrm{C}$ thermal grill stimulus in comparison to a uniform cold $\left(20^{\circ} \mathrm{C}\right)$ or warm $\left(40{ }^{\circ} \mathrm{C}\right)$ stimulus in order to analyse sex differences in the changes of sensation evoked by thermal grill stimulation. In addition, we determined sex differences in thermal detection and thermal pain threshold in 1252 undergraduate students of medicine and dentistry, by retrospectively analysing quantitative sensory testing (QST) data that had been collected in student laboratory courses. QST data included cold and warm detection thresholds at different baseline (adaptation) temperatures between 20 and $40{ }^{\circ} \mathrm{C}$. This allows further investigation of thermal sensation circuitries as earlier studies have analysed thermal detection thresholds only at baseline temperatures around the neutral/comfort zone of $32{ }^{\circ} \mathrm{C}[24,25]$.

After finding sex differences in TGI and QST data, the objective was to test the hypothesis of a sex-dependent correlation of the TGI with the subject's thermal sensitivity and/or thermal pain sensitivity. Therefore, we correlated the TGI with cold or warm sensation and, in addition, with thermal pain sensitivity.

\section{Methods}

\section{Subjects}

The thermal grill experiments were performed in a group of 136 medical undergraduate students of the Ludwig-Maximilians University Munich (58 males and
78 females, aged 20-30 years). Volunteers were recruited by signing a list available in the student laboratories. This list included all information about the purpose of the study including the aim, i.e. the investigation of sex differences in thermoreception. On the day of the experiment, the subjects gave consent to participate in the experiments.

The retrospective analysis of thermal sensation data included 12,874 records of QST measurements from 1252 students (776 females and 476 males, mean age $22 \pm 3$ years (median interquartile range)). The data were collected during neurophysiological laboratory courses for medical and dental undergraduate students at the LudwigMaximilians University Munich (Germany) in the years 2007-2011. Students were informed that data of thermal sensitivity were to be gathered from healthy subjects and that the data were later to be analysed anonymously to generate comprehensive results for instruction and publication purposes. Six or seven students of each class of around 20 students volunteered to undergo the noninvasive tests; the others performed the acquisition of data or other tasks. Volunteers gave consent to participate in the experiments before the start of the tests. The analysis was performed with the permission of the local ethics committee of the Ludwig-Maximilians University of Munich.

For analysing correlations of thermal pain threshold with the TGI, all values were determined in one experimental session for each of 40 (20 female and 20 male) medical students. The recruitment of these subjects (aged 20-30 years) who were not part of the 136 cohort was the same as described above for the thermal grill experiments. On the day of the experiment, the subjects gave consent to participate in the experiments. The whole study (all three experiments) complied with the guidelines established by the Declaration of Helsinki and was approved by the local ethics committee of the Ludwig-Maximilians University Munich for experiments involving human subjects.

\section{Equipment and experimental protocol}

The thermal grill experiments were performed by an investigator. The subjects were naïve with respect to the "illusion phenomenon". They were informed about the rating procedure and assured of the harmlessness of all stimulation parameters. For stimulation, subjects placed the palmar surface of the right hand on the thermal grill that was fixed to a table. The setup of the thermal grill device has been described elsewhere [19]. Briefly, the thermal grill consists of 15 bars (tubes) that are perfused with warm or cold water. The temperatures tested were 20,40 , and $20{ }^{\circ} \mathrm{C}$ alternating with $40{ }^{\circ} \mathrm{C}$ (thermal grill stimulus). A second thermal grill with all bars (tubes) held at $32{ }^{\circ} \mathrm{C}$ was used as a control to establish a baseline temperature of the skin immediately prior to each thermal stimulus trial. 
To examine sensations associated with grill stimulation, the hand was first placed on the $32{ }^{\circ} \mathrm{C}$ reference grill surface for $20 \mathrm{~s}$. The hand was then exposed to either a uniform $20^{\circ}$ or uniform $40{ }^{\circ} \mathrm{C}$ stimulus or the interleaved $20 / 40{ }^{\circ} \mathrm{C}$ (grill) stimulus for $20 \mathrm{~s}$. Each stimulus was presented three times with a minimum inter-stimulus interval of $5 \mathrm{~min}$. After each stimulus presentation, subjects were asked to specify their evoked perception by using the descriptors "warm/heat, cold, unpleasantness, pain, burning, stinging and prickling". Then, the subjects rated the intensities of their sensations using numeric rating scales (NRS) from 0 to 100 to rate the "thermal intensity" of (a) their cold sensation and (b) their warm sensation. The scale anchors were " $0=$ neutral" and " $100=$ worst cold or worst warm/hot", respectively, and along one side of the scale there were three additional descriptors indicating that the subject should rate intensities of perceived coldness or warmth/heat. The scale was numbered from 0 to 100 in increments of 10. In addition, subjects rated perceived pain and unpleasantness on numeric scales from 0 to 100 , also numbered from 0 to 100 in increments of 10 and with the anchors " $0=$ no pain or no unpleasantness" and " 100 = worst pain or as unpleasant as can be imagined".

QST measurements were self-performed by the students in groups of two or three. The students were informed about the procedure of the measurements but received no prior training. In each group, one student served as subject and the other(s) operated the computer and recorded the data. For stimulation, a computerized thermotest device TSA 2001-II Neurosensitive Analyser (MEDOC, Ramat Yishai, Israel) was used with a standard $30 \times 30 \mathrm{~mm}$ thermode. The method of limits was employed [26] and the rate of temperature change was $1.5{ }^{\circ} \mathrm{C} / \mathrm{s}$. The cut-off temperatures were 0 and $50{ }^{\circ} \mathrm{C}$, respectively. During the experiment, the subject was not able to see the computer screen.

Cold and warm detection thresholds (CDT and WDT, respectively) were measured at different baseline temperatures of the thermal ramp stimulus with the thermode affixed with a Velcro strip to the ventral surface of the forearm near the wrist. Table 1 shows the sequence of measurements at different baseline temperatures in the range of $20-40{ }^{\circ} \mathrm{C}$ resulting in different values of $\mathrm{CDT}_{\mathrm{X}^{\circ} \mathrm{C}}$

Table 1 Sequence of measurements at different baseline temperatures in the range of $20-40^{\circ} \mathrm{C}$

\begin{tabular}{lll}
\hline Recording sequence & $\begin{array}{l}\text { Baseline } \\
\text { temperature }\end{array}$ & $\begin{array}{l}\text { Sequence of detection } \\
\text { thresholds }\end{array}$ \\
\hline 1st & $30{ }^{\circ} \mathrm{C}$ & $\mathrm{WDT}_{30}{ }^{\circ} \mathrm{C}, \mathrm{CDT}_{30}{ }^{\circ} \mathrm{C}$ \\
2nd & $35{ }^{\circ} \mathrm{C}$ & $\mathrm{CDT}_{35}{ }^{\circ} \mathrm{C}, \mathrm{WDT}_{35}{ }^{\circ} \mathrm{C}$ \\
3rd & $25^{\circ} \mathrm{C}$ & $\mathrm{WDT}_{25}{ }^{\circ} \mathrm{C}, \mathrm{CDT}_{25}{ }^{\circ} \mathrm{C}$ \\
4th & $40{ }^{\circ} \mathrm{C}$ & $\mathrm{CDT}_{40}{ }^{\circ} \mathrm{C}, \mathrm{WDT}_{40}{ }^{\circ} \mathrm{C}$ \\
5th & $20{ }^{\circ} \mathrm{C}$ & $\mathrm{WDT}_{20}{ }^{\circ} \mathrm{C}, \mathrm{CDT}_{20}{ }^{\circ} \mathrm{C}$ \\
\hline
\end{tabular}

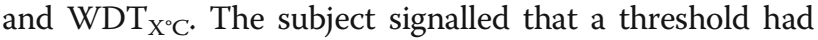
been reached by pressing a button, at which point the temperature change of the thermode was halted, the direction reversed and the temperature returned to the respective baseline temperature. Subjects were instructed to press the button as soon as they detected a change of the temperature ("Press the response button immediately when you perceive a change of the thermode temperature, i.e. warmer during WDT tests or colder during CDT tests"). Thresholds of five consecutive runs were averaged to determine $\mathrm{CDT}_{\mathrm{X}^{\circ} \mathrm{C}}$ and $\mathrm{WDT}_{\mathrm{X}^{\circ} \mathrm{C}}$.

For measuring cold and heat pain threshold (CPT and $\mathrm{HPT}$ ), the thermode was placed on the subject's skin so as to stimulate the thenar eminence. The baseline temperature was $32{ }^{\circ} \mathrm{C}$. The instruction was as follows: Indicate by pressing a button the occurrence of a painful or unpleasant sensation of heat or cold, respectively. Thresholds of five consecutive runs were averaged to determine CPT and HPT.

Data used for correlation analyses of the TGI with thermal pain threshold were obtained in an additional experiment that was carried out by an investigator. The TGI and CPT and HPT were measured in 40 subjects (20 female and 20 male medical students) who had been trained in QST measurements. The subjects were naïve with respect to the "illusion phenomenon" according to the thermal grill experiment with 136 subjects. The thermal grill and QST parameters were the same as described above.

\section{Statistical analysis}

Statistical analysis was performed using the program SPSS Statistics Version 22, IBM, Chicago, IL, USA. With respect to the thermal grill experiments, KolmogorovSmirnov test revealed that for NRS ratings of thermal sensation and for thermal threshold, data values were not normally distributed. Therefore, the median as well as the first and third quartiles (boxes) and range (error bars) were used for data description and Friedman's ANOVA with post hoc testing (Wilcoxon's signed-rank test) was employed for statistical analysis of the thermal grill-evoked sensations. To assess sex differences in the qualities of the thermal grill-evoked sensations, the Mann-Whitney $U$ test was performed. Differences in sensations evoked by the uniform cold $\left(20^{\circ} \mathrm{C}\right)$ or warm $\left(40{ }^{\circ} \mathrm{C}\right)$ stimulus and the thermal grill $\left(20^{\circ} / 40^{\circ} \mathrm{C}\right.$ interleaved) stimulus are presented as means \pm standard error of the mean (SEM; $\Delta$ values) for male and female subjects separately. For analysing sex differences in the intensities of grill-evoked sensations, Student's $t$ test for unpaired samples was performed. Linear regression analyses were carried out to evaluate the effects of thermal sensation and thermal pain sensation on the effects of the thermal grill-evoked warmth/heat and coldness. To 
assess dependency between two variables of non-normally distributed data, Spearman's correlation coefficient $(\rho)$ was calculated. A Bonferroni-type adjustment was made for multiple correlation analyses. $P$ value $<0.05$ was considered to indicate a statistically significant difference and is indicated by an asterisk (*) in the tables and figures.

To assure the quality of the retrospectively analysed QST data, the variances of all 12.874 records were analysed. The variances were distributed in the range $0.00-260.26$. The distribution of variances is characterized as follows: median 0.280 , interquartile range (IQR) 1.430, mean 2.577, and standard deviation (SD) 9.565. Skewness was reduced calculating an upper criterion (Eq. 1), following McGill and colleagues [27]

$$
\text { crit }=\text { median }+1.5 \times \mathrm{IQR}
$$

Any record with a variance above a crit value of 2.425 was excluded from further analysis. In addition, records with incomplete or implausible data were excluded, i.e. data lacking the information about sex or age or data obtained using incorrect stimulation sites. Some 9940 records remained for further analysis. Following the suggestions by Rolke and colleagues [2], all records were logarithmized (base 10) before being subjected to any statistical test. Sex differences were analysed post hoc using Tukey's honest significant difference test [28]. Analyses were performed using the language for statistical computing R [29].

For analysis of multimodal distribution of CPTs, the CPT data were fitted by a Gaussian mixed model using the R-program AdaptGauss [30] (Additional file 1). For calculating the probability density function (PDF), the median of five repetitions of the CPT test of each subject was estimated and rescaled for stimulus intensity and a log transformation was subsequently carried out. The PDF was calculated for all subjects participating in the CPT measurements $(N=296)$.

\section{Results}

\section{Thermal grill illusion (TGI)}

Thermal grill-evoked sensations were analysed in 136 subjects (78 females and 58 males). Sensations reported after contact between the palmar hand surface and the thermal grill in grill mode, i.e. with alternating 20 and $40{ }^{\circ} \mathrm{C}$ tempered bars, exhibited a characteristic thermal profile. All 136 subjects reported sensations of "warm" or "hot" in the centre of the area in contact with the skin and 41 subjects $(30 \%)$ described this sensation as painful (Table 2). All subjects (except three) reported "cold" mostly at the periphery of the contact surface (e.g. at the finger tips). The intensity of the cold sensation changed during grill stimulation in some subjects, and 10 subjects (7\%) reported no "cold" at the end of the grill stimulation.
Table 2 Sex differences in the sensations evoked by thermal grill stimulation $\left(20 / 40{ }^{\circ} \mathrm{C}\right)$

\begin{tabular}{lllll}
\hline & \multicolumn{2}{l}{$\rho(N=78)$} & \multicolumn{2}{c}{${ }^{\prime}(N=58)$} \\
\hline Sensation & $N$ & $\%$ & $N$ & $\%$ \\
Warm/heat & 78 & 100.0 & 58 & 100.0 \\
Cold & 72 & 92.3 & 54 & 93.1 \\
Unpleasantness & 54 & 69.2 & 30 & $51.7^{*}$ \\
Pain & 30 & 38.5 & 11 & $19.0^{*}$ \\
Burning & 39 & 50.0 & 18 & $32.8^{*}$ \\
Stinging & 10 & 12.8 & 10 & 17.2 \\
Prickling & 9 & 11.5 & 12 & 20.7 \\
\hline
\end{tabular}

Significant differences between sexes are marked by asterisks (Mann Whitney $U$ test)

Some 84 subjects $(65 \%)$ described the entire sensation as "unpleasant" (Table 2). When being asked to report on additional qualities of the evoked perception, the descriptors burning, stinging and prickling were chosen by 57,20 and 21 subjects (42, 15 and 15\%), respectively (Table 2). Similar thermal grill data were obtained in the additional group of 40 subjects; the data are summarized in a table (see Additional file 1: Table S1).

Pooled data of intensities of sensations arising from uniform thermal and grill stimuli are shown in Fig. 1 for all 136 subjects. In response to the uniform $40{ }^{\circ} \mathrm{C}$ stimulus all subjects reported the sensation of warm with a median intensity of 35 on the NRS. The uniform $20{ }^{\circ} \mathrm{C}$ stimulus evoked the sensation of cold in all subjects with a median intensity of 30 on the NRS. The thermal sensations of warm and cold evoked by the grill stimulus were rated with median intensities of 50 for warm (heat) and 30 for cold on the NRS. With respect to the intensity ratings, all four indicators "cold-induced cold sensation", "warm-induced warm sensation", "grill-evoked cold sensation" and "grill-evoked warm/heat sensation" differed significantly $\left(\chi^{2}=190.0, p<0.001\right.$, Friedman's ANOVA, $p<0.02$, post hoc tests using Wilcoxon's signed-rank test). Similarly, regarding the sensation of unpleasantness, the three stimulation conditions cold, warm and grill differed significantly $\left(\chi^{2}=85.5, p<0.001\right.$, Friedman's ANOVA, post hoc tests using Wilcoxon's signed-rank test). Pain was felt by only one subject on exposure to the uniform warm stimulus (35 on the NRS). Under grill stimulation, pain was rated as less intense than unpleasantness (median value of 0 versus $20, p<0.001$, Wilcoxon's signed-rank test).

Figure 1 shows numeric scale ratings of thermal sensations evoked by stimulation with the thermal grill.

\section{Sex differences in the TGI}

Subjects were asked to report on the quality of the perception evoked by the thermal grill stimulation $\left(20 / 40{ }^{\circ} \mathrm{C}\right.$ interleaved). Table 2 lists the descriptors that the subjects 


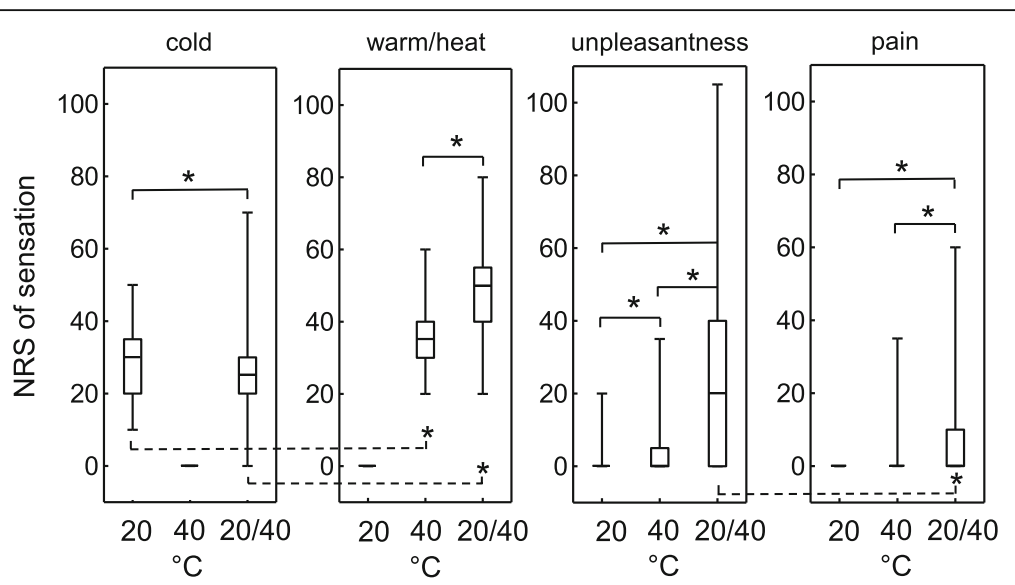

Fig. 1 Numeric scale ratings (NRS) of thermal sensations (cold, warm/ heat), unpleasantness and pain evoked by placement of the right hand on the thermal grill for $20 \mathrm{~s}$. For insets "cold" and "warm/ heat" hold " $0=$ neutral" and "100 = worst cold or worst warm/hot", respectively. For insets "pain" and "unpleasantness" hold " $0=$ no pain or no unpleasantness" and "100 = worst pain or as unpleasant as can be imagined", respectively. Three different thermal stimuli were tested: uniform $20^{\circ} \mathrm{C}$, uniform $40{ }^{\circ} \mathrm{C}$ and grill (bars tempered alternately at 20 and $40{ }^{\circ} \mathrm{C}$ ) and each stimulus induced up to four different sensations. Ratings of all subjects $(N=136)$ are shown as medians with first and third quartiles (box) and range (whiskers, i.e. capped bars). Friedman's ANOVA with post hoc testing using Wilcoxon's signed-rank test was performed and significant differences are marked by asterisks

could choose combined with the selection frequency for female and male subjects separately. Significant sex differences were observed regarding the sensations of "unpleasantness", "pain" and "burning". These sensations were described more often by females than males $(p<0.05$, Mann-Whitney $U$ test).

Females and males did not differ with respect to the rated intensities of warm or cold sensation evoked by the uniform warm or cold stimuli (see Additional file 2: Figure S1). Additionally, both sexes rated the grill stimulus as warmer and more unpleasant than the uniform warm stimulus $(p<0.001$, Wilcoxon's matched pairs test, Bonferroni's correction, Fig. 2). In order to analyse sex differences in thermal grill-evoked sensations, the changes of sensation intensities on moving from the uniform 20 or $40{ }^{\circ} \mathrm{C}$ to the grill mode $\left(20 / 40{ }^{\circ} \mathrm{C}\right.$ interleaved) were calculated. With respect to the change in warm intensity on moving from the uniform $40{ }^{\circ} \mathrm{C}$ to the grill mode, females showed greater changes than males. The difference in warm intensity between the two stimuli $40{ }^{\circ} \mathrm{C}$ and grill $(\Delta$ values) was $14.0 \pm 1.2$ versus $9.7 \pm 1.3$ for female and male subjects, respectively, $p<0.05$, Student's $t$ test, Fig. 2b). The difference in cold intensity was $-4.5 \pm 1.5$ versus $-2.5 \pm 1.6$ for female and male subjects (n.s., Student's $t$ test, Fig. 2a). The difference in the sensation of unpleasantness was $24.9 \pm 3.1$ (females)
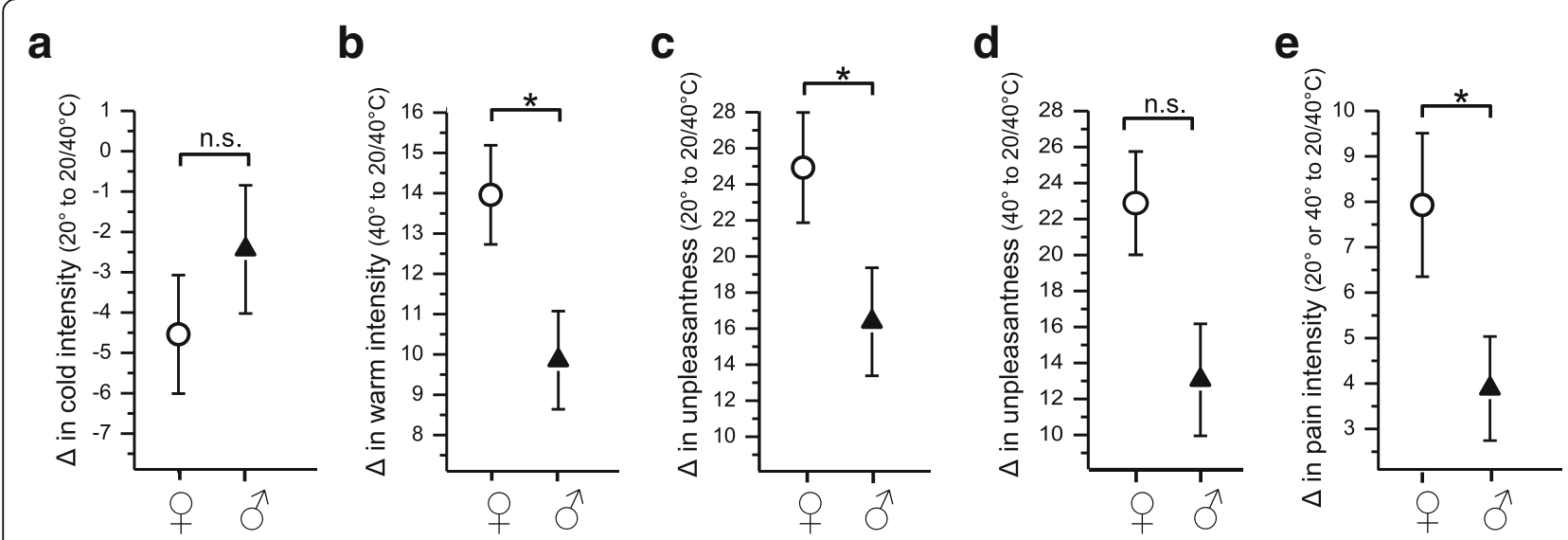

Fig. 2 The change in intensity of different sensations ( $\triangle$ values, means \pm SEM, panels a-e) occurring by moving from the uniform temperature condition $\left(20\right.$ or $40{ }^{\circ} \mathrm{C}$ ) to the thermal grill condition (20 and $40{ }^{\circ} \mathrm{C}$ interleaved). To assess sex differences, Student's $t$ test for unpaired samples was performed and significant differences are marked by asterisks. In panel e, sensations evoked by the 20 or $40{ }^{\circ} \mathrm{C}$ stimulus were pooled because stimulation with uniform temperatures did not induce any pain sensation 
versus $16.3 \pm 3.0$ (males) for the two stimulation conditions $20^{\circ} \mathrm{C}$ and grill $(p<0.05$, Student's $t$ test, Fig. $2 \mathrm{c})$ and $22.9 \pm 2.9$ (females) versus $13.0 \pm 3.1$ (males) for the two stimuli $40{ }^{\circ} \mathrm{C}$ and grill ( $p=0.05$, Student's $t$ test, Fig $2 \mathrm{~d}$ ). Sex differences in the sensation of unpleasantness, with females rating higher intensities than males, were also significant when including only the subjects who felt unpleasantness by thermal grill stimulation (data not shown). The difference in pain intensity between the two stimuli "uniform 20 or $40{ }^{\circ} \mathrm{C}$ " and grill was $7.9 \pm 1.6$ and $3.8 \pm 1.2$ for female and male subjects, respectively $(p<0.05$, Student's $t$ test, Fig. 2e). However, when including the responders of pain only, the rated intensities did not differ between the two sexes $(22.9 \pm 2.6, n=30$ and $20.0 \pm 2.8, n=11$ for females and males respectively, data not shown).

In summary, female subjects more often felt a burning sensation, unpleasantness and pain with a grill stimulus set at a $20 / 40{ }^{\circ} \mathrm{C}$ pattern than did males. In addition, females felt the grill stimulus, in comparison to the uniform cold or warm stimulus, as significantly warmer, less cold and more unpleasant than males.

Figure 2 shows sex differences in thermal grill-induced sensations.

\section{Sex differences in thermal thresholds}

To investigate sex differences in thermal detection and thermal pain threshold, a total of 9940 records from 1252 students (776 females and 476 males) were analysed. Thermal detection thresholds (CDT, WDT) were measured on the ventral surface of the forearm. Mean threshold values $\left({ }^{\circ} \mathrm{C}\right.$ from baseline) are shown in Fig. 3 and means \pm SEM are summarized in Table 3. Both CDT and WDT were dependent on the baseline (adaptation) temperature, i.e. the starting temperature of the thermal ramp stimulus. The curve of WDT plotted against the baseline temperature $\left(20-40{ }^{\circ} \mathrm{C}\right)$ showed a systematic decrease of WDT, with females showing significantly lower WDT values (smaller $\Delta$ warm values in Fig. $3 \mathrm{~b}$ ) than males over the whole range of baseline temperatures tested (Tukey's HSD, $p<0.05$, Fig. 3b, Table 3). Within each sex group, WDT was significantly different in pairwise comparisons of neighbouring baseline temperatures, except for the pair 35 vs. $40{ }^{\circ} \mathrm{C}$ (Tukey's HSD, $p<0.001$ ). In contrast, the function of CDT vs. baseline temperature showed a completely different progression, namely that of an optimum curve with the highest CDT (smallest $\Delta$ cold value in Fig. 3a) at $30{ }^{\circ} \mathrm{C}$ baseline temperature. $\mathrm{CDT}$ values ( $\Delta$ values) were significantly higher at all other baseline temperatures tested (Tukey's HSD, $p<0.001$, Fig. 3a, Table 3). This effect was independent of sex. Females had significantly lower CDT values (smaller $\Delta$ cold values) than males at all baseline temperatures tested (Tukey's HSD, $p<0.05$, Fig. 3a).

Figure 3 shows sex differences in cold and warm detection thresholds at different baseline temperatures.

CPT and HPT were measured over the thenar eminence starting at a baseline temperature of $32{ }^{\circ} \mathrm{C}$. CPT for females was significantly higher (less cold) and HPT lower than for males (Tukey's HSD, $p<0.01$ ). The median CPT was $17.7{ }^{\circ} \mathrm{C}(N=208)$ for females and $14.0^{\circ} \mathrm{C}$ $(N=88)$ for males. The median HPT was $45.9{ }^{\circ} \mathrm{C}$ $(N=333)$ for females and $48.1{ }^{\circ} \mathrm{C}(N=247)$ for males. In summary, females were more sensitive with respect to thermal detection and thermal pain thresholds than males.

Analysis showed that the distribution of CPTs was clearly multi-modal. The distribution of the $N=296 \mathrm{log}$ transformed threshold data could be described with a Gaussian mixture model composed of $M=6$ Gaussians (Fig. 4 and Additional file 3: Table S2). The first two Gaussians at $31.3^{\circ} \mathrm{C}$ (grey) and $30.4{ }^{\circ} \mathrm{C}$ (yellow), after data was retransformed from the log domain to the threshold temperatures, represent the false responses of the subjects indicating CDT instead of CPT. The modes of the

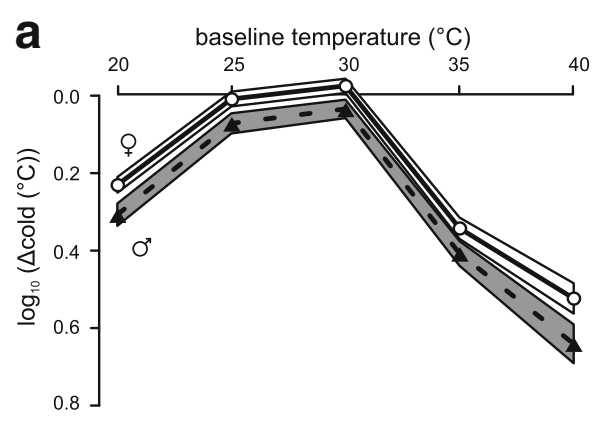

b

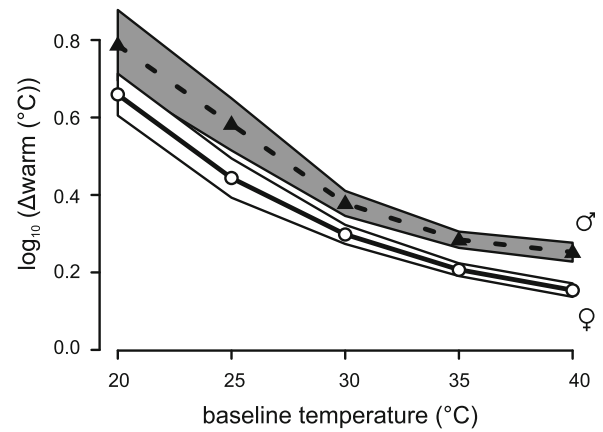

Fig. 3 Cold (a) and warm (b) detection thresholds at different baseline (adaptation) temperatures for female (ㅇ) and male ( $\left.\delta^{\wedge}\right)$ participants. Means $\left(\log _{10}\left({ }^{\circ} \mathrm{C}\right.\right.$ from baseline $) \pm 95 \%$ confidence band of the means of the detection thresholds are shown. Mean threshold values of females are presented as open circles (confidence band: white area) and means of males are presented as filled triangles (confidence band: grey area) 
Table $\mathbf{3}$ Thermal detection thresholds at different baseline temperatures

\begin{tabular}{|c|c|c|c|c|c|c|}
\hline & \multirow[t]{2}{*}{ Sex } & \multicolumn{5}{|c|}{ Baseline temperature $\left({ }^{\circ} \mathrm{C}\right)$} \\
\hline & & 20 & 25 & 30 & 35 & 40 \\
\hline \multirow[t]{2}{*}{ Warm threshold $\left({ }^{\circ} \mathrm{C}\right)$} & $q$ & $4.6 \pm 1.07 N=179$ & $2.8 \pm 1.06 N=225$ & $2.0 \pm 1.03 N=356$ & $1.6 \pm 1.02, N=364$ & $1.4 \pm 1.02 N=352$ \\
\hline & $\hat{0}$ & $6.1 \pm 1.11 N=84$ & $3.8 \pm 1.08 N=134$ & $2.4 \pm 1.04 N=212$ & $1.9 \pm 1.02 N=228$ & $1.8 \pm 1.03 N=217$ \\
\hline \multirow[t]{2}{*}{ Cold threshold $\left({ }^{\circ} \mathrm{C}\right)$} & q & $-1.7 \pm 1.02 N=338$ & $-1.0 \pm 1.02 N=356$ & $-0.9 \pm 1.02 N=375$ & $-2.2 \pm 1.03 N=321$ & $-3.3 \pm 1.05 N=253$ \\
\hline & $\hat{o}$ & $-2.0 \pm 1.03 N=191$ & $-1.2 \pm 1.03 N=227$ & $-1.1 \pm 1.03 N=238$ & $-2.6 \pm 1.04 N=202$ & $-4.4 \pm 1.06 N=135$ \\
\hline
\end{tabular}

Thermal detection thresholds are listed as ${ }^{\circ} \mathrm{C}$ from baseline (mean $\pm \mathrm{SEM}$ ) for $N$ subjects at different baseline temperatures

Gaussians \#3, \#4, \#5 and \#6 (Fig. 4: green, cyan, orange and blue curves) were obtained at 27.0, 23.0, 16.5 and $2.1{ }^{\circ} \mathrm{C}$. The Gaussians \#3, \#4 and \#5 showed significantly different modes between male and female subjects (Welch modified two-sample $t$ test, $p<0.01$; Additional file 3: Table S2).

Figure 4 shows a Gaussian mixture model of cold pain thresholds.

\section{Correlation of the TGI with thermal sensitivity}

The sensation of warm evoked by the uniform warm stimulus correlated positively with the sensation of cold evoked by the uniform cold stimulus in both sexes ( $\rho=0.26, p=0.009$ after Bonferroni's correction, data not shown) demonstrating that the thermal sensitivities to warmth and coldness are associated. Figure 5 illustrates simple linear regression results using either warm stimulus-evoked warm sensation or cold stimulus-evoked cold sensation as the only independent variable: The increase in warm sensation evoked by grill stimulation ( $\Delta$ grill heat, i.e. the difference in warm intensity between the two stimuli $40{ }^{\circ} \mathrm{C}$ and grill) correlated negatively with the warm stimulus-evoked warm sensation in males $(\rho=-0.38, p=0.006$ after Bonferroni's correction, Fig. 5b) whereas there was no significant correlation in females $(\rho=0.03$, n.s.). Thus, in male subjects $\Delta$ grill heat decreased with increasing sensitivity to warmth. $\Delta$ grill cold, i.e. the difference in cold intensity between the two stimuli $20{ }^{\circ} \mathrm{C}$ and grill, decreased with an increasing cold stimulus-evoked sensation of cold (negative correlation with $\rho=-0.30, p=0.001$ after Bonferroni's correction). This means that the higher the cold sensitivity of a subject the less cold was the sensation evoked by the grill stimulus. This effect was stronger in females than in males $(\rho=-0.36$, $p=0.001$ after Bonferroni's correction) for females versus $\rho=-0.19$, n.s. for males, Fig $5 \mathrm{a}$ ).

Figure 5 shows correlations of the thermal grill-induced changes in thermal sensations with thermal sensitivity.

\section{Correlation of the TGI with thermal pain threshold}

Consistent with the retrospective data analysis of QST measurements in 1.252 subjects, females in the group of 40 subjects showed significantly higher (less cold) CPT values than males (median $\mathrm{CPT}$ at the thenar eminence
10.4 for females versus 7.4 for males, $p<0.03$, Mann Whitney $U$ test, Additional file 1: Table S1). Regarding $\Delta$ thermal pain threshold (HPT versus CPT), females showed significant lower values than males revealing a higher thermal pain sensitivity of females compared with males $(p<0.02$, Student's $t$ test, Additional file 1 : Table S1). The TGI obtained in the group of 40 subjects was similar to the TGI found in the group of 136 subjects, although sex differences were not significant in the small group (e.g. for $\Delta$ grill cold $p=0.09$, Student's $t$ test, table in Additional file 1: Table S1).

Multiple regression analyses of $\Delta$ grill heat were carried out using CPT, HPT and $\Delta$ thermal pain threshold as the independent variables. Including all 40 subjects of both sexes showed that $\Delta$ grill heat correlated significantly with CPT $(\rho=0.44, p=0.03$ after Bonferroni's correction). Simply stated, within individual subjects, the higher, i.e. the less cold the CPT, the more intense the grill-induced increase of warm/heat sensation. Thus, females showing higher CPT than males showed a stronger grill heat sensation (Fig. 6b). Multiple regression analysis of $\Delta$ grill cold with CPT, HPT and $\Delta$ thermal pain threshold as the independent variables showed no significant dependency of $\Delta$ grill cold with any of these variables. Sex differences in $\Delta$ grill cold were found with respect to the slopes of the linear regressions. With both, increasing $\mathrm{CPT}$ and decreasing $\Delta$ thermal pain threshold, $\Delta$ grill cold decreased in females while it increased in males (Fig. 6a, e). This means that females described the grill stimulus as increasingly less cold while males described it as decreasingly less cold with higher cold pain sensitivity or higher thermal pain sensitivity, respectively.

Figure 6 shows correlations of the thermal grillinduced changes in thermal sensations with thermal pain thresholds.

\section{Discussion}

The present study demonstrates that females show a stronger TGI than males, since females more often feel a burning sensation, unpleasantness and pain and describe the grill stimulus as significantly warmer and less cold than males. In addition, the study demonstrates that females show a higher thermal sensitivity and thermal 


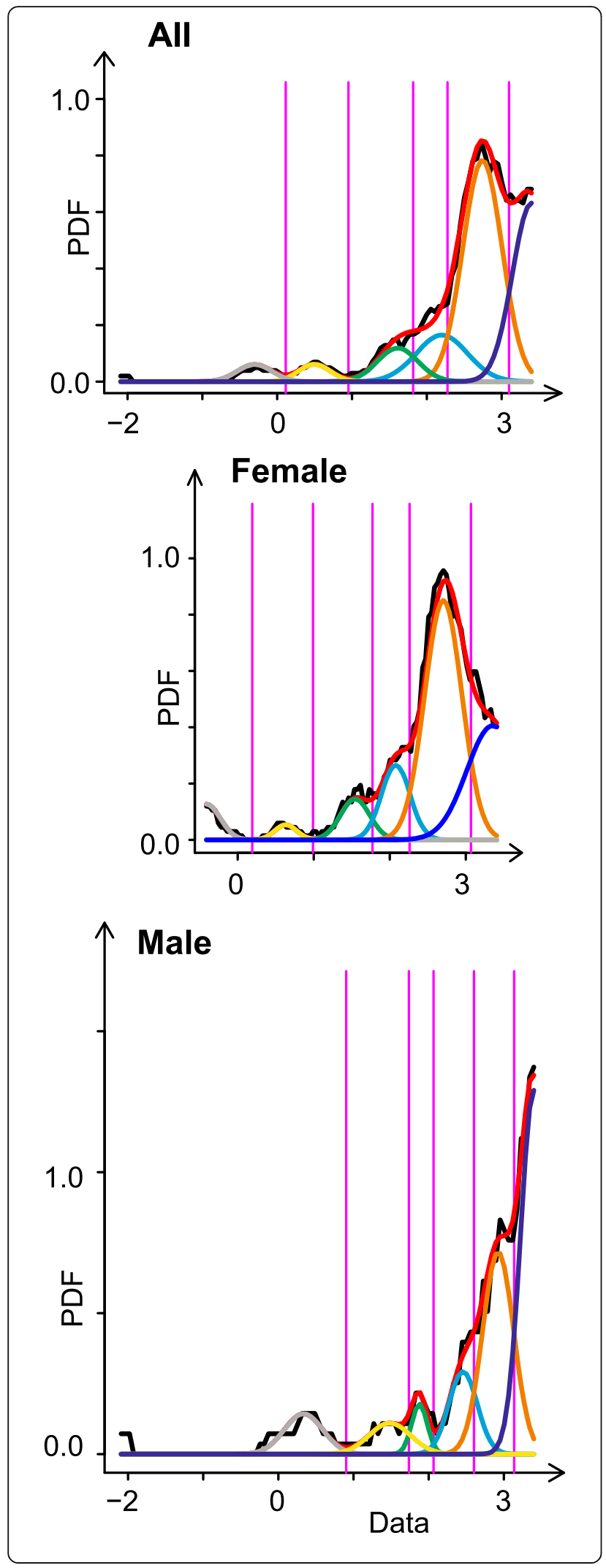

Fig. 4 Gaussian mixture model of cold pain thresholds. The graphs display the data after rescaling for stimulus intensity and subsequent log transformation. The density distributions are presented as probability density function (PDF), estimated using a Gaussian mixture model (R-program AdaptGauss [30]). The optimum number of mixes was found to be $M=6$. Black curve: PDF; red curve: Gaussian mixed model, Gaussians: grey $=1$, yellow $=2$, green $=3$, cyan $=4$, orange $=5$, blue $=6$; purple: Bayes boundaries of the Gaussians. ALL: $N=296$ subjects, FEMALE: $N=208$, MALE: $N=88$

pain sensitivity than males. The stronger TGI in females correlates with the higher sensitivity to cold and cold pain in females compared with males.

\section{Sex differences in the TGI and thermal sensitivity}

In the present study thermal grill stimulation $\left(20 / 40{ }^{\circ} \mathrm{C}\right.$ interleaved) of the hand induced a unique perception including sensations of warmth, coldness, unpleasantness, burning, stinging, prickling and pain. Consistent with the literature, the present study shows the TGI to be very complex [10-23]. A novel finding in the present study was that the TGI is also sex-dependent. Regarding thermal sensitivity and thermal pain sensitivity, we also found sex differences in the present study. With respect to detection thresholds, our data are consistent with earlier studies $[4,7]$. We found that the mean detection threshold of females and males differed by $0.2-0.4{ }^{\circ} \mathrm{C}$, with females reporting higher (less cold) CDT values and lower (less warm) WDT values than males, indicating thermal sensitivity is higher in females than in males (see Table 3 and Fig. 3). The sex difference in WDT was more pronounced at low skin temperatures, e.g. $1.5{ }^{\circ} \mathrm{C}$ for $\mathrm{WDT}_{20}{ }^{\circ} \mathrm{C}$ and $\mathrm{WDT}_{25}{ }^{\circ} \mathrm{C}$ (see Fig. 3b), a new finding that reveals clear sex differences in warm detection threshold at slightly cool $\left(25^{\circ} \mathrm{C}\right)$ or cold $\left(20^{\circ} \mathrm{C}\right)$ skin temperatures thus implying sex differences in the complex processing of "cold" and "warm" inputs in thermal perception. For thresholds of cold pain and heat pain, females showed lower HPT and higher (less cold) CPT values in the present study which is consistent with the literature, albeit for CPT data published findings are somehow contradictory $[2-4,6,8]$.

With respect to the distribution of CPT data, we found a sex-dependent multimodal distribution in the present study that is similar to the CPT data distribution published recently by Lötsch and colleagues [31]. Fitting a Gaussian mixture model to the log-transformed CPT data revealed three Gaussian distributions with modes at 23, 17 and $2{ }^{\circ} \mathrm{C}$ (see Fig. 4). According to Lötsch and colleagues [31], the localization of the first and second Gaussians may be interpreted as reflecting the contribution of the TRPM8 receptor that starts to respond at $24{ }^{\circ} \mathrm{C}$ [32] and the TRPA1 receptor that starts to sense cold at $17{ }^{\circ} \mathrm{C}$ [33]. Sex differences were found for these 

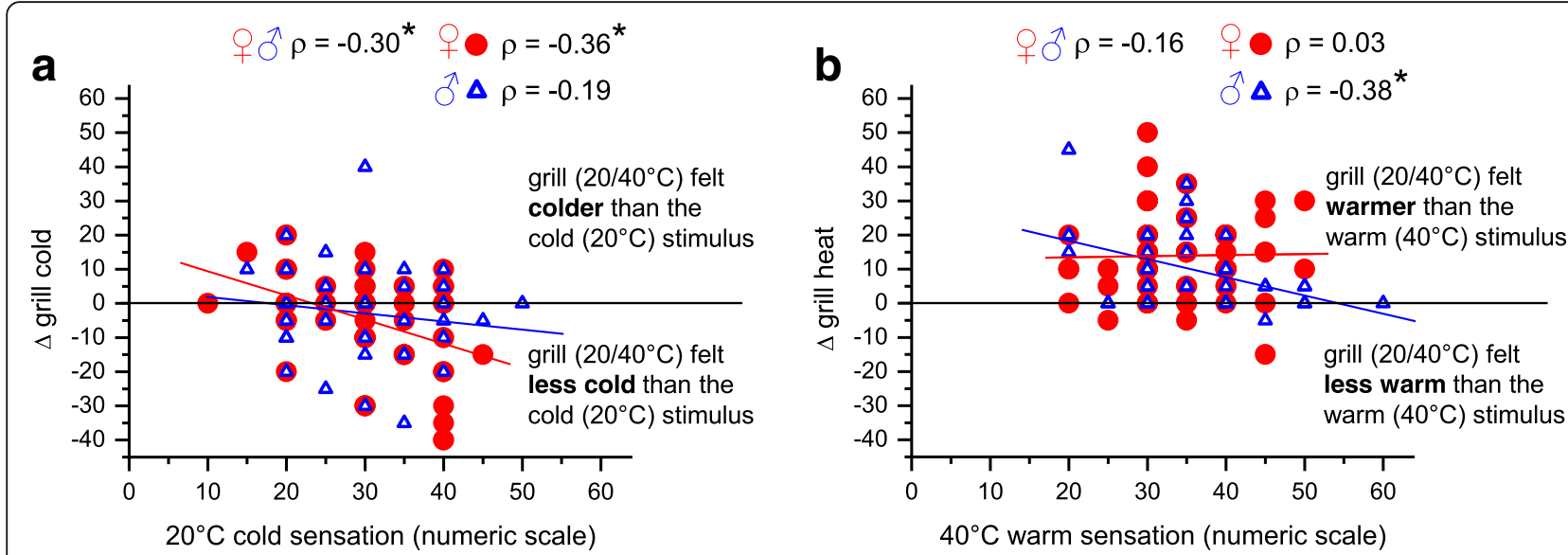

Fig. 5 The correlation between $\Delta$ grill cold, i.e. the change in cold intensity from the $20^{\circ} \mathrm{C}$ to the thermal grill condition $\left(20 / 40^{\circ} \mathrm{C}\right)$ and the intensity of cold during the $20^{\circ} \mathrm{C}$ condition (a). The correlation between the $\Delta$ grill warm, i.e. the change in warm intensity from the $40^{\circ} \mathrm{C}$ to the thermal grill condition $\left(20 / 40^{\circ} \mathrm{C}\right.$ ) and the intensity of warmth during the $40^{\circ} \mathrm{C}$ condition (b). A positive number on the $y$-axis indicates an increase in cold or warm sensation during the thermal grill stimulation compared to the uniform cold $\left(20^{\circ} \mathrm{C}\right)$ or warm $\left(40^{\circ} \mathrm{C}\right)$ stimulus, respectively. A negative number indicates the opposite, i.e. a decrease. Data of male $(N=58)$ and female $(N=78)$ subjects are presented separately using different symbols and colours. As values of thermal intensity ratings were not normally distributed (Kolmogorov-Smirnov test), Spearman's correlation coefficient $\rho$ was calculated. $P<0.05$ after Bonferroni's correction was considered significant and this is indicated by asterisks

Gaussians in the present study indicating sexdependent receptor characteristics at the skin area where the cold stimuli had been applied. For the Gaussian with mode at $2{ }^{\circ} \mathrm{C}$, a sex-dependent difference of response probability was found (female $15 \%$, male $32 \%$, respectively (see Additional file 3: Table S2) indicating that other temperature-sensing receptors, e.g. TRPC5, KCNK2, ASIC2 or ASIC3, might show sex-dependent spatial densities in the skin as well. The mode at $27{ }^{\circ} \mathrm{C}$ may be seen as the earliest response due to an uncomfortable cold stimulus and the modes at 31.3 and $30.4{ }^{\circ} \mathrm{C}$ as false responses of untrained students who indicated the CDT instead of the CPT.

Thermal sensitivity as a function of the baseline temperature Our retrospective analysis of sex-dependent thermal threshold data provides the novel finding that warm and cold detection thresholds are seen to be affected by the baseline (adaptation) temperature. Studies to date have addressed thermal detection thresholds at baseline temperatures around the neutral/comfort zone, usually $32{ }^{\circ} \mathrm{C}$, which is approximately the mean skin temperature at standard ambient temperature [24, 25]. Our data demonstrate that, independent of sex, the CDT as a function of the baseline temperature has the form of an optimum curve with the optimum in the range $25-30{ }^{\circ} \mathrm{C}$. In humans, innocuous skin temperatures of cold are signalled by cold-sensitive A $\delta$ fibres [13, 34]. Recently, a micro-neurography study in humans has shown that the response rate of an $\mathrm{A} \delta$ fibre to a staircase cold stimulation has the form of an optimum curve with the maximum response rate at $26{ }^{\circ} \mathrm{C}$ baseline temperature and lower response rates at lower or higher baseline temperatures (see Fig. 7 in [34]. Hence, our CDT data (see Fig. 3a) might be explained by $A \delta$ fibre activation. In addition, the activity of $\mathrm{C} 2$ fibres, a population of $\mathrm{C}$ fibres responding to warming and innocuous cooling [34] is likely to play a role. $\mathrm{C} 2$ fibre activity is inhibited by $\mathrm{A} \delta$ fibre input; when the A $\delta$ fibre input is blocked experimentally, innocuous cooling becomes painful $[35,36]$, probably by disinhibition of $\mathrm{C} 2$ fibre activity. Thus, the interplay between $\mathrm{A} \delta$ and C2 fibre activity during skin cooling is likely to depend on the baseline (adaptation) temperature of the skin. In contrast, the curves of WDT vs. baseline temperature show a hyperbolic form with the lowest WDT at $40{ }^{\circ} \mathrm{C}$ and the highest at $20^{\circ} \mathrm{C}$ (see Fig. 3b) confirming data of early studies [37, 38]. According to the literature, warm fibres in primates respond to warm stimuli above $30{ }^{\circ} \mathrm{C}$ only [38-42], conversely there is no evidence for warm receptor activation by warm stimuli at baseline temperatures below $30{ }^{\circ} \mathrm{C}$. Thus, the mechanism of warm detection at cool skin temperatures remains unclear. At cool skin temperatures, $\mathrm{A} \delta$ and $\mathrm{C} 2$ fibres are activated. During subsequent warming of the skin, activation decreases for $\mathrm{A} \delta$ fibres and increases for $\mathrm{C} 2$ fibres. This may lead to a sensation of declining cold and thus to the subjects' reaction to denote their WDT.

\section{Correlation of the TGI with cold sensitivity and cold pain sensitivity}

A model proposed to explain the TGI, albeit excluding the non-painful sensations, indicates that thermal grill stimulation with interleaved warm and cold stimuli reduces the cold signal that is responsible for inhibiting 

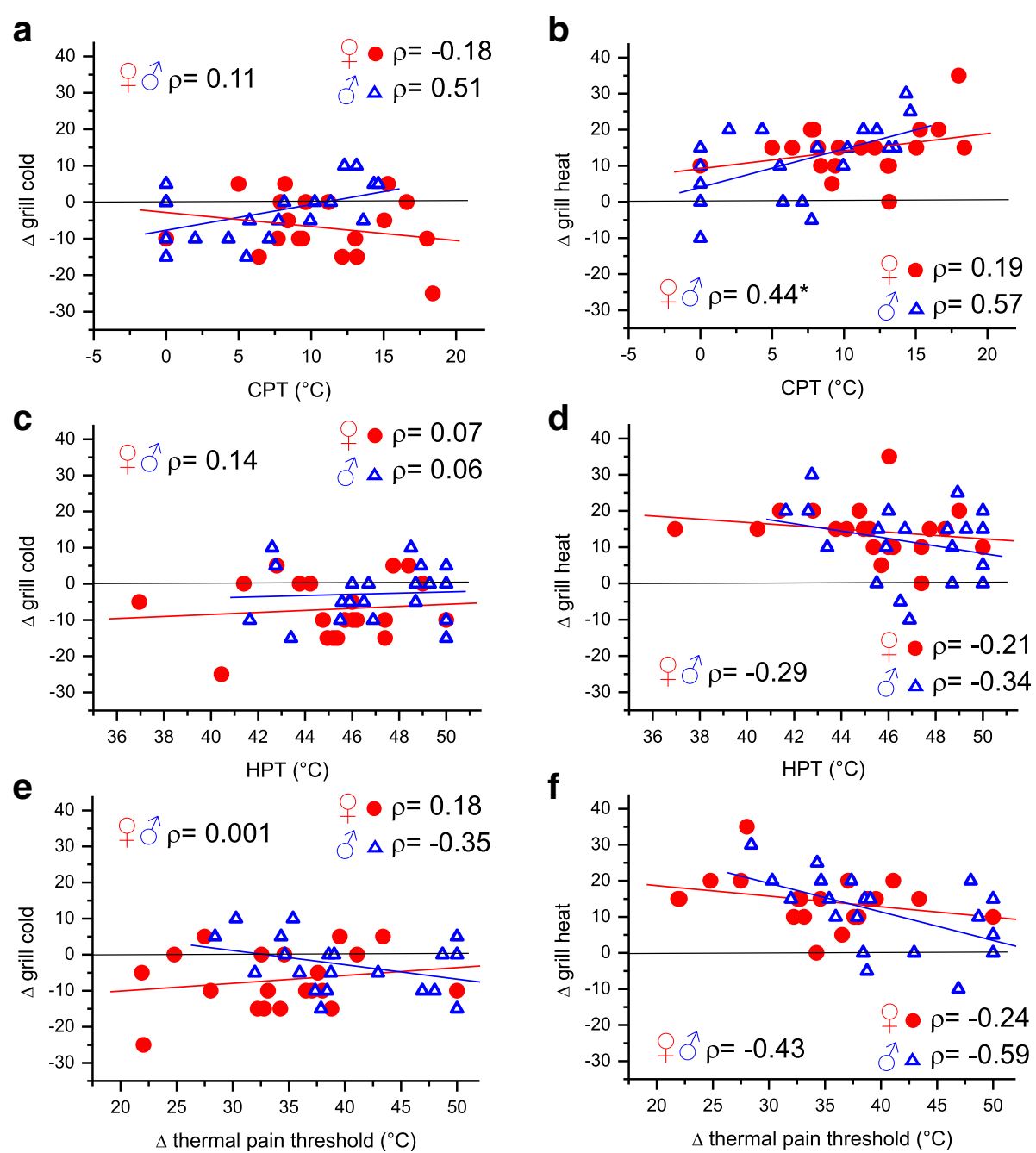

Fig. 6 Linear regressions of $\Delta$ grill heat (grill-induced increase in warm/heat sensation, panels $\mathbf{b}$, $\mathbf{d}$ and $\mathbf{f}$ ) and $\Delta$ grill cold (grill-induced increase in cold sensation, panels $\mathbf{a}, \mathbf{c}$ and $\mathbf{e}$ ) with either CPT, HPT or $\triangle$ thermal pain threshold (HPT versus CPT) as the only dependent variable. Data from male $(N=20)$ and female $(N=20)$ subjects are presented separately using different symbols and colours. As the thermal threshold values were not normally distributed (Kolmogorov-Smirnov test) Spearman's correlation coefficient ( $\rho$ ) was calculated to assess dependency between two variables. $P<0.05$ after Bonferroni's correction was considered significant and this is indicated by asterisks

the activity in central multi-modal neurons responsive to heating, pinch and cold (HPC), hence leading to disinhibition and consequently by increasing the magnitude of the HPC signal to pain [10]. The assumption that a strong cold signal is jointly responsible for the TGI is supported by the recent finding that topical application of menthol, an activator of the cold signal, leads to an enhanced grill-evoked heat sensation [19], probably by enhancing the disinhibition of the HPC signal. Consistent with this is the finding of the present study that both cold and cold pain sensitivity are related to the intensity of the TGI (see Figs. 5 and 6), e.g. the stronger the individual sensation of cold under the uniform cold stimulus, the larger was the decrease of cold sensation under grill conditions in the present study (see Fig. 6a). In contrast, warm and heat pain sensitivity were not related to the thermal grill-evoked sensations in the present study showing that the "warm" input is less important for the TGI (see Fig. 6b).

Recent publications have reported that higher sensitivity to cold and heat pain is associated with a stronger TGI, e.g. stronger sensations of unpleasantness and pain $[18,43]$. The reverse effect has been found in studies with patients with psychiatric disorders showing that a lower sensitivity to cold and heat pain is accompanied by a less intense TGI compared with controls [16, 44]. Thus, sex differences in the thermal grill sensations are assumed to be related to sex differences in heat and cold pain. Our correlation analysis (see Figs. 5 and 6) shows that the sexdependent sensitivity to cold and cold pain is responsible 
for the sex-dependent TGI. It is primarily the decrease of cold sensation under grill conditions that differs between the two sexes. In the present study, the reduction of cold sensation under grill stimulation increased with higher $\mathrm{CPT}$ in females but decreased in males (see Fig. 6a). Thus, the females' higher sensitivity to cold pain is related to the grill-evoked sensation of less cold in females compared with males. In addition, the present study shows that the higher the non-painful cold sensitivity of a subject the less cold was the grill-evoked sensation and this effect was stronger in females than in males (see Fig. 5a and Discussion above). The females' grill-evoked sensation-warmer and more unpleasant (and if so burning and painful) than the males' grill-evoked sensation-is supported by the females' sensation of less cold, which is dependent on the sensitivity to cold and cold pain.

\section{Limitations}

It has to be taken into account that the retrospective data analysis was performed on QST data collected by selfperformed measurements by students who were only informed but not trained in the measurement procedure. This fact may reduce the validity of the test. When comparing thermal pain threshold of the retrospectively analysed data with thermal pain threshold obtained in additional QST measurements that were carried out by an investigator in a group of students who were trained in QST measurements, we found similar values for HPT and colder values for CPT in trained versus untrained subjects (see "Sex differences in thermal thresholds" and Additional file 1: Table S1). The less cold CPT in untrained than in trained subjects may be due to the untrained subjects indicating the CDT instead of the CPT in the experiment (see Fig. 4 and Additional file 3: Table S2). Another possibility is that trained subjects may wait a little longer before "confirming" their sensation of cold pain than untrained subjects. Regarding sex differences in CPT, females and males differed independent of training. A limitation of the present study is the fact that a female investigator tested the TGI and the thermal pain threshold in the small cohort of 40 subjects. This may bias psychophysical outcomes in relation to sex. For painful stimuli, male subjects reportedly show weaker responses when the investigator is female rather than male $[45,46]$. Another limitation of the present study is the fact that different stimulation sites were used to determine thermal detection and thermal pain threshold in the retrospectively analysed QST measurements (ventral surface of the forearm near the wrist and thenar eminence, see 2.2). However, the stimulation sites including the hand stimulated in the thermal grill experiments are unlikely to differ markedly; in an earlier study, thermal detection and pain threshold and the TGI were found to be similar for the volar forearm and the palm of the hand [19].

\section{Conclusions}

Our study provides further evidence for a strong interaction between the thermoreceptive and nociceptive systems. The new aspect of the retrospective data analysis of our study is the finding that thermal detection threshold not only differs between the two sexes but is also dependent on the baseline temperature. A specific progression of an optimum curve was found for the function relating $\mathrm{CDT}$ to baseline temperature with the highest sensitivity of cold detection around the basal skin temperature implying a complex processing of "cold" and "warm" inputs in thermal perception. The multimodal distribution of the cold pain thresholds indicates sex-dependent differences in response characteristics and spatial densities of the cold receptors TRPA1 and TRPM8 in the skin. Our findings regarding the TGI, first, that the TGI was more pronounced in females and, second, that the intensity of the illusion correlated with the subjects' sensitivity to cold and cold pain leads to the assumption that sex differences in the cold-related thermoreception are responsible for sex differences in the TGI. For further investigation, it would be of interest to test the sex dependency of the TGI under a strong activation of the cold signal, e.g. by the application of cold receptor agonists corresponding to TRPA1 and TRPM8.

\section{Additional files}

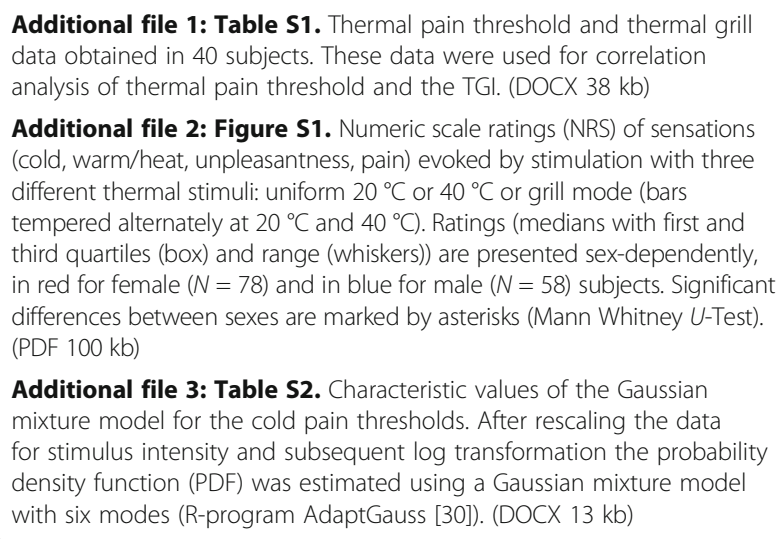

Additional file 2: Figure S1. Numeric scale ratings (NRS) of sensations (cold, warm/heat, unpleasantness, pain) evoked by stimulation with three different thermal stimuli: uniform $20^{\circ} \mathrm{C}$ or $40^{\circ} \mathrm{C}$ or grill mode (bars tempered alternately at $20^{\circ} \mathrm{C}$ and $40^{\circ} \mathrm{C}$ ). Ratings (medians with first and third quartiles (box) and range (whiskers)) are presented sex-dependently, in red for female $(N=78)$ and in blue for male $(N=58)$ subjects. Significant differences between sexes are marked by asterisks (Mann Whitney $U$-Test). (PDF 100 kb)

Additional file 3: Table S2. Characteristic values of the Gaussian mixture model for the cold pain thresholds. After rescaling the data for stimulus intensity and subsequent log transformation the probability density function (PDF) was estimated using a Gaussian mixture model with six modes (R-program AdaptGauss [30]). (DOCX 13 kb)

\section{Abbreviations \\ CDT: Cold detection threshold; CPT: Cold pain threshold; HPC: Heating, pinch, cold; HPT: Heat pain threshold; IQR: Interquartile range; NRS: Numeric rating scale; QST: Quantitative sensory testing; SEM: Standard error of the mean; TGI: Thermal grill illusion; WDT: Warm detection threshold}

\section{Acknowledgements}

We would like to thank Franz Rucker for his efforts in constructing the thermal grill device (Averbeck et al. [19]). We are very grateful to John Davis and Peter Grafe for their support throughout the project and would like to thank them for comments on the manuscript and for improving the English. 


\section{Availability of data and materials}

The datasets generated and/or analysed during the current study are available from the corresponding author on reasonable request.

\section{Authors' contributions}

$\mathrm{BA}, \mathrm{DK}$ and FK conceived and designed the experimental setup and the experiments. BA and DK wrote the manuscript. Quantitative sensory testing of the subjects was carried out in the students' laboratory courses and data were analysed by LS, DK and FK. Testing of subjects with the thermal grill device as well as testing of the 40 subjects applying QST and thermal grill stimulation was done by BA and data were analysed by BA. Statistical analyses were carried out by BA and DK. All authors read and approved the final manuscript.

\section{Ethics approval and consent to participate}

The analysis was performed with the permission of the local ethics committee of the Ludwig-Maximilians University of Munich (reference number 150-14).

\section{Consent for publication}

Not applicable.

\section{Competing interests}

The authors declare that they have no competing interests.

\section{Publisher's Note}

Springer Nature remains neutral with regard to jurisdictional claims in published maps and institutional affiliations.

\section{Author details}

${ }^{1}$ Department of Physiology, University of Munich, Munich, Germany. ${ }^{2}$ Institute of Human Movement Science and Health, Faculty of Behavioral and Social Science, Chemnitz University of Technology, Chemnitz, Germany. ${ }^{3}$ Department of Physiology, Biomedical Center Munich (BMC), University of Munich, Planegg-Martinsried, D-82152, Germany.

\section{Received: 17 January 2017 Accepted: 1 August 2017} Published online: 01 September 2017

\section{References}

1. Sarlani E, Farooq N, Greenspan JD. Gender and laterality differences in thermosensation throughout the perceptible range. Pain. 2003;106:9-18.

2. Rolke R, Magerl W, Campbell KA, Schalber C, Caspari S, Birklein F, Treede RD. Quantitative sensory testing: a comprehensive protocol for clinical trials. Eur J Pain. 2006;10:77-88.

3. Hashmi JA, Davis KD. Noxious heat evokes stronger sharp and annoying sensations in women than men in hairy skin but not in glabrous skin. Pain. 2010;151:323-9. doi:10.1016/j.pain.2010.06.026.

4. Kuhtz-Buschbeck JP, Andresen W, Göbel S, Gilster R, Stick C. Thermal perception and nociception of the skin: a classic paper of Bessou and Perl and analyses of thermal sensitivity during a student laboratory exercise. Adv Physiol Educ. 2010;34:25-34. doi:10.1152/advan.00002.2010.

5. Racine $M$, Tousignant-Laflamme $Y$, Kloda LA, Dion D, Dupuis $G$, Choinière $M$. A systematic literature review of 10 years of research on sex/gender and pain perception - part 1: are there really differences between women and men? Pain. 2012;153:619-35. doi:10.1016/j.pain.2011.11.025.

6. Horn ME, Alappattu MJ, Gay CW, Bishop M. Fear of severe pain mediates sex differences in pain sensitivity responses to thermal stimuli. Pain Res Treat. 2014;897953 doi:10.1155/2014/897953.

7. Golja P, Tipton MJ, Mekjavica IB. Cutaneous thermal thresholds: the reproducibility of their measurements and the effect of gender. J Thermal Biol. 2013;28:341-6. doi:10.1016/S0306-4565(03)00010-X.

8. Waller R, Smith AJ, O'Sullivan PB, Slater H, Sterling M, McVeigh JA, Straker LM. Pessure and cold pain threshold reference values in a large, young adult, pain-free population. Scand J Pain. 2016;13:114-22. doi:10.1016/j. sjpain.2016.08.003.

9. Alrutz S. On the temperature senses: II. The sensation 'hot'. Mind. 1898;7:141-4.

10. Craig $A D$, Bushnell MC. The thermal grill illusion: unmasking the burn of cold pain. Science. 1994:265:252-5.

11. Bouhassira D, Kern D, Rouaud J, Pelle-Lancien E, Morain F. Investigation of the paradoxical painful sensation ('illusion of pain') produced by a thermal grill. Pain. 2005;114:160-7.
12. Leung AY, Wallace MS, Schulteis G, Yaksh T. Qualitative and quantitative characterization of the thermal grill. Pain. 2005;116:26-32.

13. Defrin R, Benstein-Sheraizin A, Bezalel A, Mantzur O, Arendt-Nielsen L. The spatial characteristics of the painful thermal grill illusion. Pain. 2008;138:577-86. doi:10.1016/j.pain.2008.02.012

14. Bach $P$, Becker $S$, Kleinböhl D, Hölzl R. The thermal grill illusion and what is painful about it. Neurosci Lett. 2011;505:31-5. doi:10.1016/j.neulet.2011.09.061.

15. Boettger MK, Schwier C, Bär KJ. Sad mood increases pain sensitivity upon thermal grill illusion stimulation: implications for central pain processing. Pain. 2011;152:123-30. doi:10.1016/j.pain.2010.10.003.

16. Boettger MK, Grossmann D, Bär KJ. Thresholds and perception of cold pain, heat pain, and the thermal grill illusion in patients with major depressive disorder. Psychosom Med. 2013;75:281-7. doi:10.1097/PSY. ob013e3182881a9c.

17. Lindstedt F, Johansson B, Martinsen S, Kosek E, Fransson P, Ingvar M. Evidence for thalamic involvement in the thermal grill illusion: an FMRI study. PLoS One. 2011; doi:10.1371/journal.pone.0027075.

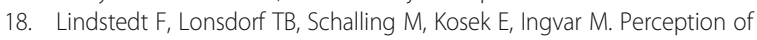
thermal pain and the thermal grill illusion is associated with polymorphisms in the serotonin transporter gene. PLoS One. 2011; doi:10.1371/journal.pone. 0017752.

19. Averbeck B, Rucker F, Laubender RP, Carr RW. Thermal grill-evoked sensations of heat correlate with cold pain threshold and are enhanced by menthol and cinnamaldehyde. Eur J Pain. 2013;17:724-34. doi:10.1002/j. 1532-2149.2012.00239.

20. Adam F, Alfonsi P, Kern D, Bouhassira D. Relationships between the paradoxical painful and nonpainful sensations induced by a thermal grill. Pain. 2014;155:2612-7. doi:10.1016/j.pain.2014.09.026.

21. Harper DE, Hollins M. Coolness both underlies and protects against the painfulness of the thermal grill illusion. Pain. 2014;155:801-7. doi:10.1016/j. pain.2014.01.017.

22. Hunter J, Dranga R, van Wyk M, Dostrovsky JO. Unique influence of stimulus duration and stimulation site (glabrous vs. hairy skin) on the thermal grillinduced percept. Eur J Pain. 2015;19:202-15. doi:10.1002/ejp.538.

23. Alfonsi P, Adam F, Bouhassira D. Thermoregulation and pain perception: evidence for a homoeostatic (interoceptive) dimension of pain. Eur J Pain. 2016;20:138-48. doi:10.1002/ejp.717.

24. Jakovljević M, Mekjavić IB. Reliability of the method of levels for determining cutaneous temperature sensitivity. Int J Biometeorol. 2012;56: 811-21. doi:10.1007/s00484-011-0483-9.

25. Selim MM, Wendelschafer-Crabb G, Hodges JS, Simone DA, Foster SX, Vanhove GF, Kenned WR. Variation in quantitative sensory testing and epidermal nerve fibre density in repeated measurements. Pain. 2010;151: 575-81. doi:10.1016/j.pain.2010.06.034.

26. Yarnitsky D, Sprecher E, Zaslansley R, Hemli JA. Heat pain thresholds: normative data and repeatability. Pain. 1995;60:329-32.

27. McGill R, Tukey JW, Larsen WA. Variations of box plots. Am Stat. 1978:32:12-6.

28. Tukey JW. Comparing individual means in the analysis of variance. Biometrics Vol. 1949:5:99-114.

29. R Core Team R. A language and environment for statistical computing. Vienna: R Foundation for Statistical Computing; 2015

30. Ultsch A, Thrun MC, Hansen-Goos O, Lötsch J. Identification of molecular fingerprints in human heat pain thresholds by use of an interactive mixture model R toolbox (AdaptGauss). Int J Mol Sci. 2015;16:25897-911. doi:10. 3390/ijms161025897.

31. Lötsch J, Dimova V, Lieb I, Zimmermann M, Oertel BG, Ultsch A. Multimodal distribution of human cold pain thresholds. PLoS One. 2015;10:e0125822. doi:10.1371/journal.pone.0125822.

32. McKemy DD, Neuhausser WM, Julius D. Identification of a cold receptor reveals a general role for TRP channels in thermosensation. Nature. 2002;416:52-8.

33. Story GM, Peier AM, Reeve AJ, Eid SR, Mosbacher J, Hricik TR, Earley TJ, Hergarden AC, Andersson DA, Hwang SW, Mclntyre P, Jegla T, Bevan S, Patapoutian A. ANKTM1, a TRP-like channel expressed in nociceptive neurons, is activated by cold temperatures. Cell. 2003;112:819-29.

34. Campero M, Baumann TK, Bostock H, Ochoa JL. Human cutaneous C-fibres activated by cooling, heating and menthol. J Physiol. 2009;587:5633-52. doi: 10.1113/jphysiol.2009.176040

35. Fruhstorpher $\mathrm{H}$. Thermal sensibility changes during ischemic nerve block. Pain. 1984;20:355-61.

36. Yarnitsky D, Ochoa JL. Release of cold-induced burning pain by block of cold-specific afferent input. Brain. 1990;113:893-902. 
37. Kenshalo DR, Nafe JP, Brooks B. Variations in thermal sensitivity. Science. 1961;134:104-5

38. Kenshalo DR. Correlations of temperature sensitivity in man and monkey, a first approximation. In: Zotterman Y, editor. Sensory functions of the skin in primates with special reference to man, Wenner-Gren center international symposium series. Oxford: Pergamon Press Oxford; 1976. p. 305-30.

39. Konietzny F, Hensel H. Letters and notes: warm fibre activity in human skin nerves. Eur J Physiol/Pflügers Arch. 1975;359:265-7.

40. Konietzny $F$, Hensel $H$. The dynamic response of warm units in human skin nerves. Eur J Physiol/Pflügers Arch. 1977;370:111-4.

41. Darian-Smith I, Johnson KO, LaMotte C, Shigenaga Y, Kenins P, Champness P. Warm fibres innervating palmar and digital skin of the monkey: responses to thermal stimuli. J Neurophysiol. 1979:42:1297-315.

42. Johnson KO, Darian-Smith I, LaMotte C, Johnson B, Oldfield S. Coding of incremental changes in skin temperature by a population of warm fibres in the monkey: correlation with intensity discrimination in man. J Neurophysiol. 1979:42:1332-53

43. Schaldemose EL, Horjales-Araujo E, Svensson P, Finnerup NB. Altered thermal grill response and paradoxical heat sensations after topical capsaicin application. Pain. 2015;156:1101-11. doi:10.1097/j.pain. 0000000000000155 .

44. Bekrater-Bodmann R, Chung BY, Richter I, Wicking M, Foell J, Mancke F, Schmahl C, Flor H. Deficits in pain perception in borderline personality disorder: results from the thermal grill illusion. Pain. 2015;156:2084-92. doi: 10.1097/j.pain.0000000000000275.

45. Levine FM, De Simone LL. The effects of experimenter gender on pain report in male and female subjects. Pain. 1991;44:69-72.

46. Gijsbers $\mathrm{K}$, Nicholson F. Experimental pain thresholds influenced by sex of experimenter. Percept Mot Skills. 2005;101:803-7. doi:10.2466/pms.101.3.803-807.

\section{Submit your next manuscript to BioMed Central} and we will help you at every step:

- We accept pre-submission inquiries

- Our selector tool helps you to find the most relevant journal

- We provide round the clock customer support

- Convenient online submission

- Thorough peer review

- Inclusion in PubMed and all major indexing services

- Maximum visibility for your research

Submit your manuscript at www.biomedcentral.com/submit

) Biomed Central 